\title{
Inhibition of osteosarcoma cell progression by MacroH2A via the downregulation of cyclin $D$ and cyclin-dependent kinase genes
}

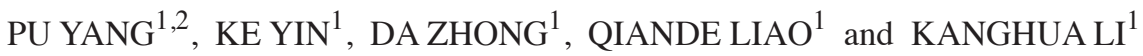 \\ ${ }^{1}$ Department of Orthopedic Surgery, The Xiangya Hospital of Central South University, Changsha, Hunan 410008; \\ ${ }^{2}$ Department of Orthopedic Surgery, The Changsha Central Hospital, Changsha, Hunan 410004, P.R. China
}

Received January 4, 2014; Accepted October 20, 2014

DOI: $10.3892 / \mathrm{mmr} .2014 .2903$

\begin{abstract}
MacroH2A is a histone modification factor the activity of which has been acutely studied in cancer progression, and a number of studies have shown that the progression of certain types of cancer is under regulation by MacroH2A. However, information regarding the underlying molecular mechanisms of MacroH2A inhibition on the cell cycle remains elusive, and elucidating this process may aid in the production of novel treatment strategies. The aim of the current study was to investigate the inhibitory effects of MacroH2A on osteosarcoma cell progression, and the possible molecular mechanisms of this process. MacroH2A overexpression and interference vectors were designed and transfected into U2-OS osteosarcoma cells. The cells underwent reverse transcription-quantitative polymerase chain reaction (RT-qPCR), western blot analysis and immunofluorescence assays. The apoptosis rate and cell cycle stage were assayed using flow cytometry. The results revealed that the overexpression of MacroH2A inhibited the progression of U2-OS osteosarcoma cells, and the cells were arrested at the $\mathrm{G}_{2} / \mathrm{M}$ stage of the cell cycle. The molecular mechanism by which MacroH2A suppresses the cell progression involves the inhibition of the expression of cyclin D and cyclin-dependent kinase (CDK) genes, including cyclin D1, cyclin D2, CDK4, CDK6 and CDK8. Taken together, the present results revealed that $\mathrm{MacroH} 2 \mathrm{~A}$ is an important modifier of chromatin that downregulates the progression of osteosarcoma cells and triggers disturbance of the cell cycle via the downregulation of cyclin D and CDK genes.
\end{abstract}

\section{Introduction}

A number of genetic and epigenetic factors have been regarded as cancer pathogen (1). A previous study of cancer epigenetics

Correspondence to: Dr Qiande Liao, Department of Orthopedic Surgery, The Xiangya Hospital of Central South University, 87 Xiangya Road, Changsha, Hunan 410008, P.R. China

E-mail: liaoqiandexy@163.com

Key words: MacroH2A, osteosarcoma, cell progression, cyclin D, cyclin-dependent kinase offered clues as to how DNA-modification, including DNA methylation, histone variants or miRNA varation, may be involved in the pathogenesis of certain types of cancer (2). Histone modification causes changes in the chromatin, and subsequently this chromatin unfolds and remodels, altering the control of gene transcription by affecting the approaching areas of the transcription start site (3). In addition, changes in chromatin influence the progression of cancer, including the occurrence, metastasis, invasion and prognosis (4). The molecular mechanisms of histone variation affect gene expression, which is associated with tumor progression and differentiation (5).

Histone variants have been regarded to have a crucial role in the regulation of nuclear activation regulation by changing chromatin structure $(6,7)$. However, the underlying mechanisms of this process are yet to be determined. Two predominant histone variants, $\mathrm{H} 3$ and $\mathrm{H} 2 \mathrm{~A}$, have been established as participants in tissue-restricted protein expression (8). Similar to its counterpart, the H2A isoform MacroH2A has a crucial role in the transformation of these variants. MacroH2A is the only histone with a tripartite structure of the N-terminal histone-fold, which contains an unstructured linker domain and a unique $\mathrm{C}$-terminal Macro domain $(9,10)$. This unique structure makes MacroH2A one of the most frequent alterations among all the histone variants $(11,12)$. Furthermore, MacroH2A has been shown to have an important role in tumor pathogenesis and development via the modification of chromatin condensation and regulation of gene expression (13). Through gene knockdown, it has been determined that MacroH2A promotes cancer development via upregulation of the cyclin-dependent protein kinase 8 (CDK8) signaling pathway. It has been established that CDK8 belongs to the CDK family and is involved in the regulation of the cell cycle $(14,15)$. The results of these studies indicate that MacroH2A affects tumor progression through changes to the cell cycle process. However, until now, the effects of MacroH2A on cell cycle regulatory genes have not been well illustrated.

In normal cells, the cell cycle processes of cell division and proliferation are strictly regulated. The key mediators of the cell cycle, CDKs and cyclins, regulate the transition of the cell cycle (16-18). During this process, dysfunctional expression of CDKs and/or cyclins, induced by abnormal metabolic activity or environmental factors, may trigger arrest or delay checkpoints in cell division $(19,20)$. Furthermore, CDKs and cyclins have important roles in stabilizing the genome in the $\mathrm{S}$ phase $(21,22)$. As a result, abnormal expression of CDKs 
and cyclins may induce the disorganization of cell proliferation, which results in the dysfunction of cell division $(23,24)$. The aim of the present study was to investigate the effects of MacroH2A on U2-OS osteosarcoma cells, by inducing MacroH2A overexpression, interference, overexpression rescue and interference rescue, and subsequently detecting the cell cycle progression using flow cytometry. Furthermore, the regulation of cyclin $\mathrm{D}$ and $\mathrm{CDK}$ expression levels by MacroH2A was investigated.

\section{Materials and methods}

Cell culture. The U2-OS acute osteosarcoma cell line was purchased from the American Type Culture Collection (Manassas, VA, USA). The cells were maintained in Dulbecco's modified Eagle's medium (Gibco-BRL, Grand Island, NY, USA), supplemented with $10 \%$ fetal bovine serum (FBS; Invitrogen Life Technologies, Carlsbad, CA, USA) at $37^{\circ} \mathrm{C}$ in a humidified incubator containing $5 \% \mathrm{CO}_{2}$. Following cell culture, the cell viability was analyzed by trypan blue exclusion. The cells were grown on glass cover slips for detection of cell morphology and were observed using an inverted microscope (Leica DMI3000B, Wetzlar, Germany).

Vector design and transfection. To examine the biophysical properties of the U2-OS cell line following knockdown and overexpression of MacroH2A, interference and overexpression vectors were created. The MacroH2A shRNA recombinant expression plasmid was purchased from Santa Cruz Biotechnology, Inc. (Dallas, TX, USA; sc-62576-SH), and the MacroH2A recombinant expression plasmid was produced in-house as follows: the open reading frame fragment was obtained from OriGene Technologies (SC110068; Rockville, MD, USA) and cloned into the plasmid between the XhoI and BamHI sites of a pcDNA3.1(t) plasmid (Invitrogen Life Technologies) to obtain the pcDNA3.1(t)-MacroH2A recombinant plasmid. Once the plasmids has been obtained, the cells were transfected with pcDNA3.1(t)-MacroH2A and/or MacroH2A shRNA Plasmid using Lipofectamine ${ }^{\mathrm{TM}} 2000$ (Invitrogen Life Technologies) according to the manufacturer' instructions. The cells were collected for analysis following a 24-h incubation period at $37^{\circ} \mathrm{C}$.

Following culture, the cells were divided into five groups (with five parallel experiments performed for each group), including a control (non-treated) group, a MacroH2A interference group (transfected with $1 \mu \mathrm{g}$ MacroH2A shRNA Plasmid), a MacroH2A overexpression group (1 $\mu \mathrm{g}$ pcDNA3.1(t)-MacroH2A Plasmid transfection), a MacroH2A interference rescue group (transfected with $1 \mu \mathrm{g}$ MacroH2A shRNA Plasmid transfection for $12 \mathrm{~h}$ followed by transfection with $1 \mu \mathrm{g}$ pcDNA3.1(t)-MacroH2A Plasmid for $12 \mathrm{~h}$ ) and a MacroH2A overexpression rescue group (transfected with $1 \mu \mathrm{g}$ pcDNA3.1(t)-MacroH2A for $12 \mathrm{~h}$ followed by transfection with $1 \mu \mathrm{g}$ MacroH2A shRNA Plasmid for $12 \mathrm{~h}$ ).

Reverse transcription-quantitative polymerase chain reaction $(R T-q P C R)$. To measure the change in the expression of mRNA transcripts in the treatment groups, RT-qPCR was performed. Total RNA was isolated from the cultured cells using TRIzol ${ }^{\circledR}$ reagent (Invitrogen Life Technologies) according to the manufacturer's instructions. A total of $1 \mu \mathrm{g}$ total RNA was transcribed to cDNA using a Takara cDNA Library Construction kit (Takara Biotechnology Co., Ltd., Dalian, China) according to the manufacturer's instruction. The transcription levels of MacroH2A, IGF-1, cyclin D1, cyclin D3, cyclin A, CDK4, CCNA2, CDC25A, CDK and CDK8 were quantified with an ABI 7500 realtime PCR system (Applied Biosystems, Inc., Foster City, CA, USA). The following primers were used: Forward: 5'-CGTTCAAGTACCGGATCAGC-3' and reverse: 5'-CAACTGCCAGCAAGATGTGT-3' for MacroH2A, forward: 5'-CTGGAGATGTATTGCGCACC-3' and reverse: 5'-AGACTTGCTTCTGTCCCCTC-3' for IGF1, forward: 5'-CCGTCCATGCGGAAGATC-3' and reverse: 5'-GAAGACCTCCTCCTCGCACT-3' for Cyclin D1, forward: 5'-AACTGACCGGGAGATCAAGG-3' and reverse: 5'-TGGCTTCAGATCTCGGTGAA-3' for Cyclin D3, forward: 5'-AGTGTGAGAGTCCCCAATGG-3' and reverse: 5'-CCTTGATCTCCCGGTCAGTT-3' for CDK4, forward: 5'-TGTTTCAGCTTCTCCGAGGT-3' and reverse: 5'-AGACTTCGGGTGCTCTGTAC-3' for CDK6, forward: 5'-TGGTCACGTCTACAAAGCCA-3' and reverse: 5'-AGCCACACCTTCCTATCAGC-3' for CDK8, and forward: 5'-AACAGCGACACCCATCCTC-3' and reverse: 5'-CATACCAGGAAATGAGCTTGACAA-3' for GAPDH. These primers for these genes were designed by Applied Biosystems, Inc., and span at least one intron/exon border to eliminate genomic DNA amplification. GAPDH was used as an endogenous control. qPCR was performed as follows: $95^{\circ} \mathrm{C}$ for $10 \mathrm{~min}$ followed by 40 cycles of $95^{\circ} \mathrm{C}$ for $15 \mathrm{sec}$ and $60^{\circ} \mathrm{C}$ for $45 \mathrm{sec}$, using the TaqMan ${ }^{\circledR}$ Universal PCR Master Mix (Applied Biosystems, Inc.). The $2^{-\Delta \Delta C t}$ method was used to analyze relative mRNA expression.

Western blot analysis. Following incubation, the cells from each group were collected by centrifugation at $200 \mathrm{x} \mathrm{g}$ for $5 \mathrm{~min}$ at $4^{\circ} \mathrm{C}$ and washed three times with ice-cold phosphate-buffered saline (PBS; Invitrogen Life Technologies). The cell pellets were resuspended in radioimmunoprecipitation assay lysis buffer (Pierce, Rockford, IL, USA) and proteins were obtained from the lysate by centrifugation. Proteins were separated by $10 \%$ SDS-PAGE and transferred to polyvinylidene fluoride membranes (Millipore, Billerica, MA, USA). Once blocked with $4 \%$ non-fat milk, the membranes were incubated with the following antibodies, purchased from Abcam (Cambridge, MA, USA): monoclonal anti-MacroH2A (ab83782), anti-cyclin D1 (ab7958), anti-cyclin D3 (ab112034), anti-CDK4 (ab7955), anti-CDK6 (ab151247) and anti-CDK8 (ab123940) and anti-GAPDH (ab9485). Subsequently, horseradish peroxidase-conjugated anti-mouse IgG secondary antibody was added to the membranes and incubated for $1 \mathrm{~h}$ at room temperature. The protein bands were detected using enhanced chemiluminescent substrate (ECL plus, GE Healthcare Life Sciences, Pittsburgh, PA, USA). Images were acquired using a Fujifilm FLA 5,000 image reader (Fujifilm, Valhalla, NY, USA).

Immunofluorescence. U2-OS osteosarcoma cells $\left(4 \times 10^{4}\right)$ were grown on sterile glass cover slips and fixed for $30 \mathrm{~min}$ in $4 \%$ paraformaldehyde solution (Sigma-Aldrich St. Louis, MO, USA) in phosphate buffer. Following incubation with blocking 
A

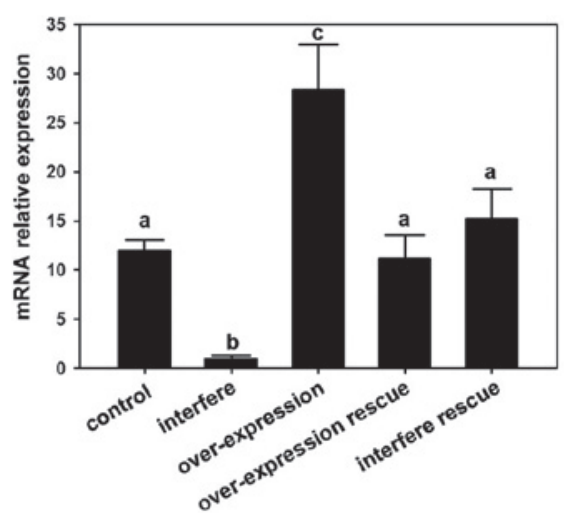

B

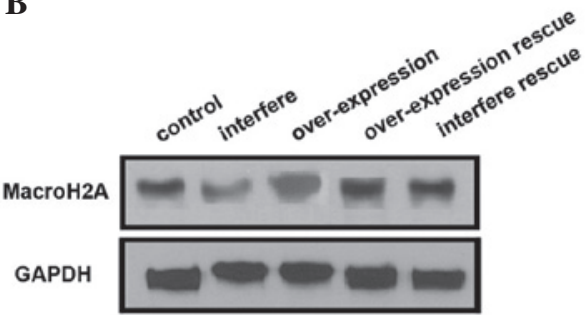

C
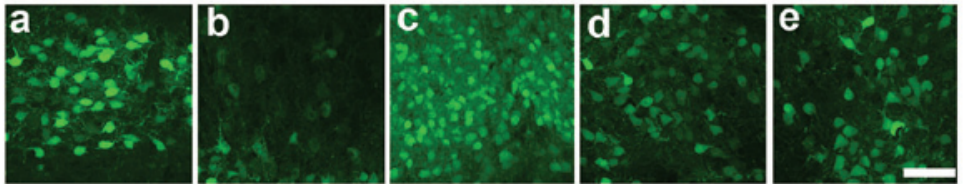

Figure 1. Interference and overexpression of MacroH2A in U2-OS osteosarcoma cells. (A) MacroH2A mRNA expression analyzed by reverse transcription-quantitative polymerase chain reaction. (different characters show significant difference, $\mathrm{P}<0.05$ ). (B) MacroH2A protein expression analyzed by western blot analysis. (C) MacroH2A protein expression assayed by immunofluorescence. (a) Negative control, (b) MacroH2A interference, (c) MacroH2A overexpression, (d) MacroH2A overexpression rescue, (e) MacroH2A interference rescue. ${ }^{\mathrm{a} N o}$ significant difference, ${ }^{b} \mathrm{P}<0.05$ and ${ }^{\mathrm{c}} \mathrm{P}<0.005$ compared with control group.

A

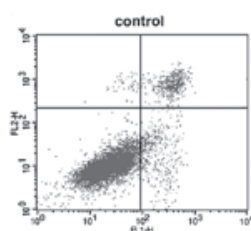

B
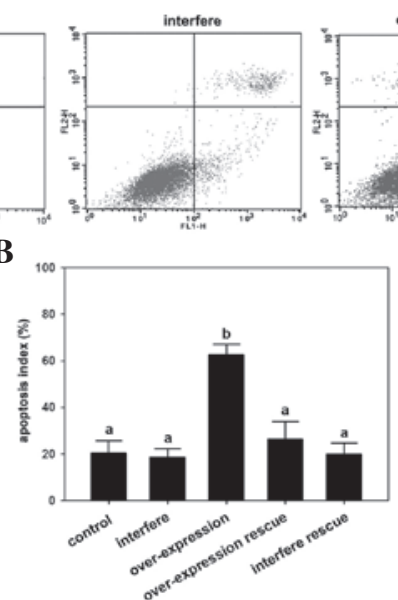

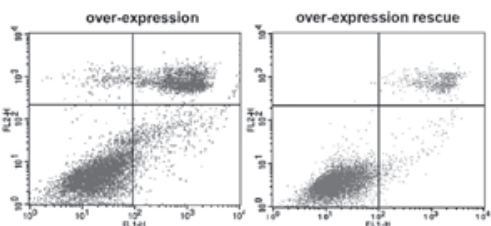

C

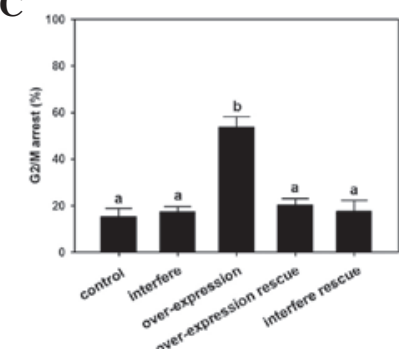

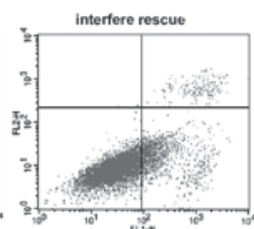

,

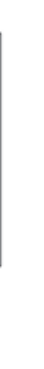

Figure 2. Regulation of osteosarcoma cell line U2-OS progression by MacroH2A among all the treatment groups. (A) Apoptosis analysis by flow cytometry. (B) Statistical analysis results of apoptosis index. (C) Statistical analysis results of $\mathrm{G}_{2} / \mathrm{M}$ arrest rates. ${ }^{\mathrm{a}} \mathrm{No}$ significant difference and ${ }^{\mathrm{b}} \mathrm{P}<0.05$ compared with control group.

reagent (5\% FBS in PBS) for $30 \mathrm{~min}$, the glasses were washed with PBS and incubated with primary antibodies overnight at $4^{\circ} \mathrm{C}$. The following primary antibodies were used: MacroH2A rabbit polyclonal, 1:1,000 dilution, $40 \mathrm{kDa}$ (ab83782); Cyclin D1 rabbit polyclonal, 1:1,000 dilution, $33 \mathrm{kDa}$ (ab7958); Cyclin D3 rabbit polyclonal, 1:1,000 dilution, 33kDa (ab112034); CDK4 rabbit polyclonal, 1:500 dilution, 34kDa (ab7955), CDK6 rabbit polyclonal, 1:1500 dilution, 37kDa (ab151247); CDK8 rabbit polyclonal, 1:500 dilution, 53kDa (ab1123940), GAPDH rabbit polyclonal, 1:5,000 dilution, 37kDa (ab9485), all obtained from Abcam. The immunofluorescence assays were performed by incubating cover slips with fluorescein isothiocyanate-conjugated secondary antibodies (F5262; Sigma-Aldrich). A conventional fluorescence microscope (Carl Zeiss, Oberkochen, Germany) was used for visualization.
Statistical analysis. Data are expressed as the mean \pm standard deviation (SD). The statistical significance of the results was analyzed using the Student's t-test or one-way analysis of variance test. $\mathrm{P}<0.05$ was considered to indicate a statistically significant difference. All analyses were performed using SPSS version 13.0 (SPSS Inc., Chicago, IL, USA).

\section{Results}

Overexpression and transfer of MacroH2A in the U2-OS osteosarcoma cell line. To detect the effect of MacroH2A on osteosarcoma cells, interference and overexpression MacroH2A vectors were constructed and transfected into osteosarcoma cells using Lipofectamine ${ }^{\mathrm{TM}}$ 2000. The mRNA expression level was analyzed by RT-qPCR. Among all of the 
A
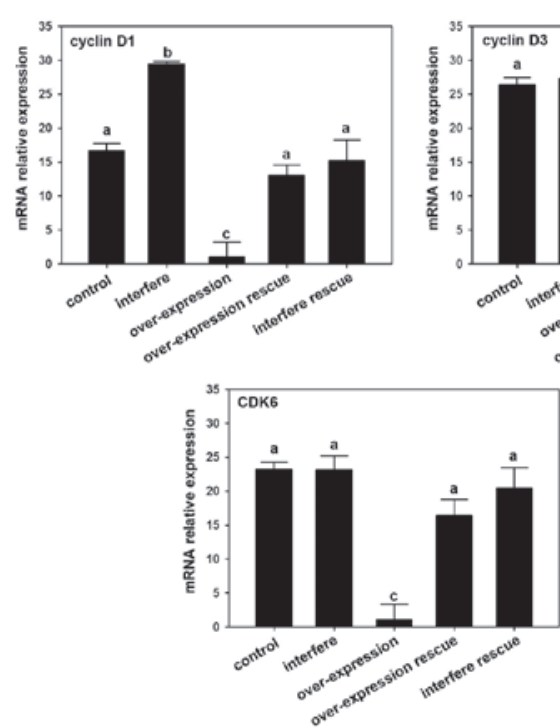
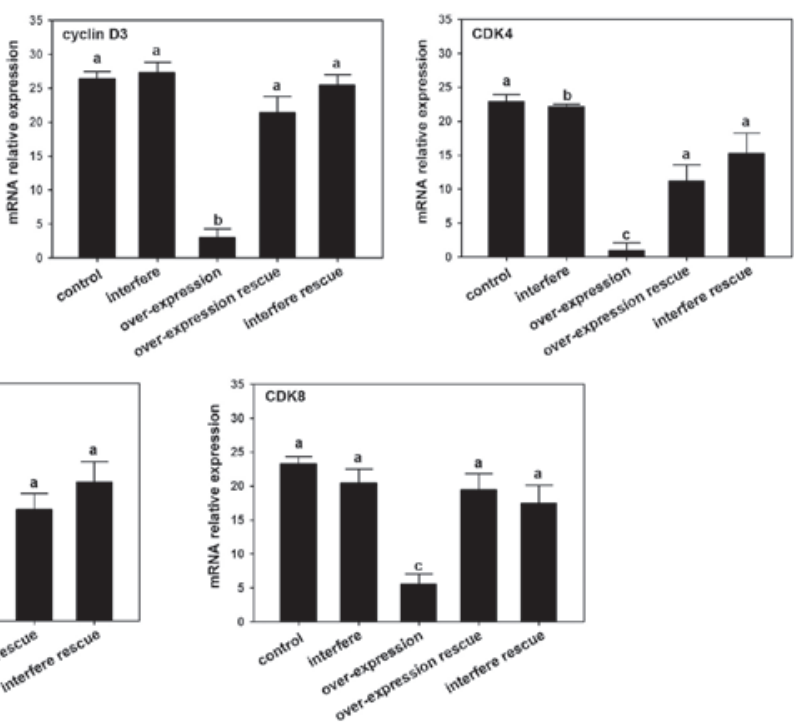

B

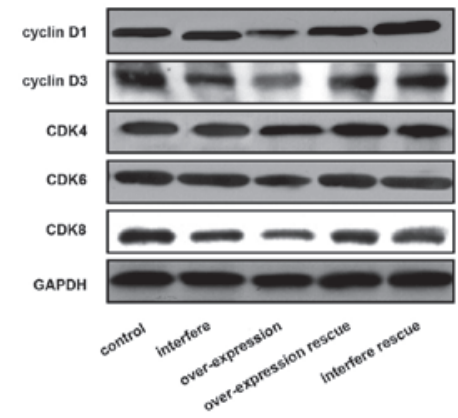

Figure 3. MacroH2A regulates cyclin D and CDK in the osteosarcoma cell line. (A) mRNA expression of cyclin D1, cyclin D3, CDK4, CDK6 and CDK8 analyzed by quantitative polymerase chain reaction (different characters showed significant difference, $\mathrm{P}<0.05$ ). (B) Protein expression of cyclin D1, cyclin D3, CDK4, CDK6 and CDK8 analyzed by western blot analysis. CDK, cyclin-dependent kinase. ${ }^{a}$ No significant difference, ${ }^{b} \mathrm{P}<0.05$ and ${ }^{\mathrm{c}} \mathrm{P}<0.005$ compared with control group.

groups, the highest MacroH2A mRNA expression level was in the MacroH2A overexpression group, while the control, $\mathrm{MacroH} 2 \mathrm{~A}$ overexpression rescue and $\mathrm{MacroH} 2 \mathrm{~A}$ interference rescue groups showed median expression. The MacroH2A interference group had the lowest MacroH2A mRNA expression levels (Fig. 1A). Furthermore, the protein expression was detected by western blot. Accordingly, the interference group had the lowest MacroH2A protein expression level compared with those of the other groups. The overexpression group had the highest MacroH2A protein density signals among all the groups. Through the use of immunofluorescence analyses, the protein expression levels were shown to have similar expression differentiation to that observed by western blotting.

Regulation of osteosarcoma cell line progression by MacroH2A. To elucidate the effects of MacroH2A on cellular regulation, the cell cycle progression and cell apoptosis levels were detected by flow cytometry. The results revealed that the highest apoptosis rate occurred in the overexpression group compared with those of the other groups $(\mathrm{P}<0.05$; Fig. $2 \mathrm{~A}$ and $\mathrm{B})$. Furthermore, the overexpression group showed a significantly higher $\mathrm{G}_{2} / \mathrm{M}$ arrest rate compared with the other groups of U2-OS osteosarcoma cells $(\mathrm{P}<0.05$; Fig. 2C).
MacroH2A regulates cyclin D and CDK expression in osteosarcoma cells. Through assaying the expression of cyclin D and CDK following interference or overexpression of MacroH2A, the mechanism of MacroH2A-induced osteosarcoma cell line inhibition was demonstrated. The results showed that expression levels of cyclin D1 and cyclin D2 were downregulated by the overexpression of MacroH2A mRNA and protein (Fig. 3). The expression level of cyclin D1 was highest in the MacroH2A interference group compared with those of the other groups, while the expression level of cyclin D2 in the MacroH2A interference group showed no significant difference compared with the control and two rescue groups (Fig. 3). In addition, the level of expression of CDK mRNA in the MacroH2A overexpression group was significantly reduced compared with the other groups. The results of western blotting also revealed that levels of the CDK4, CDK6 and CDK8 proteins in the MacroH2A overexpression group were decreased compared with the other groups (Fig. 3).

\section{Discussion}

The present study demonstrated the effect of MacroH2A on the progression of the U2-OS osteosarcoma cell line through the interference and/or overexpression of MacroH2A and 
subsequent rescue experiments. The results revealed that the treatments were efficient at artificially regulating the MacroH2A expression, and that MacroH2A suppressed the progression of U2-OS cells. Similar results have previously been reported in other types of cancers, including testicular, ovarian, lung, bladder, cervical, breast, colorectal and endometrial cancers (25-30). In these types of cancer, a low expression level of MacroH2A was found, which may be associated with cancer initiation and development. A study on melanoma indicated that the MacroH2A expression levels are strongly negative correlated with tumor development (13). Dardenne et al (31) illustrated that the alternative splicing of MacroH2A also promoted tumor progression in breast cancer cells.

Malignant tumor cells are characterized by metastasis and invasion, which is based on cell cycle progression. It has been established that $\mathrm{MacroH} 2 \mathrm{~A}$ has a central role in this process. In addition, MacroH2A maintains genomic stabilization during the replication of the genome by preventing DNA damage (9). A lack of MacroH2A may upregulate CDK8 expression levels, which contributes to cell apoptosis by blocking premature mitotic entry (13). The results of the current study indicated that the overexpression of MacroH2A triggers apoptosis in U2-OS osteosarcoma cells. Thus, the low expression of MacroH2A in tumor cells seems to be a protective effect against apoptosis. Kapoor et al (13) demonstrated that the upregulation of MacroH2A expression suppressed the progression of melanoma cells by controlling the expression of the cell cycle factor CDK8. In addition, the current study demonstrated that following the overexpression of $\mathrm{MacroH} 2 \mathrm{~A}$, the apoptosis rate of U2-OS osteosarcoma cells was increased significantly, while no such changes were observed in the other groups. In addition, the arrest stages during these cell cycles were analyzed. The results revealed that the MacroH2A overexpression group showed a significantly higher stagnancy at the $\mathrm{G}_{2} / \mathrm{M}$ stage, which indicates that the overexpression of MacroH2A in U2-OS osteosarcoma cells causes $\mathrm{G}_{2} / \mathrm{M}$ phase arrest. These results suggest that $\mathrm{MacroH} 2 \mathrm{~A}$ may trigger apoptosis by controlling the cell cycle in U2-OS osteosarcoma cells. In addition, these changes show that MacroH2 A expression blocks the process by which the divided cells pass through the $\mathrm{S}$-phase into the $\mathrm{G}_{2}$ phase during mitosis. In summary, these results demonstrate that during mitosis, an increased expression of MacroH2A arrests osteosarcoma cells at the functional $\mathrm{G}_{2} / \mathrm{M}$ stage, which blocks the cell cycle transition into mitosis, leading to cell apoptosis and preventing the metastasis and invasion tumor cells. These results provide insight into possible novel therapeutic strategies for the treatment of osteosarcoma. MacroH2A-induced inhibition of U2-OS osteosarcoma cells may be caused by its regulation of cell cycle factors. Thus, the current study subsequently addressed the regulation of cell cycle factors by MacroH2A.

To understand the molecular mechanisms behind the MacroH2A-induced apoptosis of osteosarcoma cells and the cell cycle, the expression levels of a number of cell cycle factors was investigated, including cyclin D1, cyclin D3, CDK4, CDK6 and CDK8 gene. The results showed that all five genes had a significantly lower expression level in the MacroH2A overexpression group compared with those of the other groups. Cyclin D1 has been reported to be upregulated in a number of types of cancer, and is thus regarded as an oncogene $(32,33)$. Furthermore, cyclin D1 may affect molecular process in cancer progression, including translocation, amplification and stabilization of mRNA. Additionally, cyclin D3 is a key factor in the accumulation and progression of cancer cells (34). In the present study, it was revealed that by upregulating $\mathrm{MacroH} 2 \mathrm{~A}$ expression, the expression of cyclin D1 and cyclin D3 could be suppressed. The CDK family, including CDK2, CDK3, CDK4, CDK5, CDK6, CDK7 and CDK8, also has a crucial role in the cell cycle (35). Among these family members, CDK4, CDK6 and CDK8 are the most critical in the cell cycle, controlling DNA synthesis at the beginning of the cell cycle and progressing cells from the $\mathrm{G}_{1}$-phase into the $\mathrm{S}$-phase (36). Several types of cancer have been determined to have a higher expression level of CDKs. This attribute may be a potential target in the cancer therapeutic strategy. In the current study, it was demonstrated that MacroH2A downregulates the expression of CDK4, CDK6 and CDK8 in U2-OS osteosarcoma cells. Hence, we propose that by upregulating the levels of MacroH2A in osteosarcoma cells, the expression of cyclin D and CDK may be suppressed, hence inhibiting the progression of the osteosarcoma cells.

In conclusion, the results of the present study indicate that the overexpression of MacroH2A induces apoptosis in U2-OS osteosarcoma cells and arrests the cells at the $\mathrm{G}_{2} / \mathrm{M}$-phase boundary of the cell cycle. In addition, it was determined that a high expression level of MacroH2A suppresses cell cycle progression. The underlying molecular mechanism involves the downregulation of cyclin D1, cyclin D3, CDK4, CDK6 and CDK8 genes by MacroH2A overexpression. Therefore, the expression of MacroH2A maintains a regular cell cycle by controlling the cyclin D and CDKs genes. This study has elucidated the association between chromatin structure modification and the cell cycle in osteosarcoma cells, which may offer a novel insight into potential treatment strategies for osteosarcoma.

\section{References}

1. Portela A and Esteller M: Epigenetic modifications and human disease. Nat Biotechnol 28: 1057-1068, 2010.

2. Baylin SB and Jones PA: A decade of exploring the cancer epigenome - biological and translational implications. Nat Rev Cancer 11: 726-734, 2011.

3. Kulaeva OI, Gaykalova DA and Studitsky VM: Transcription through chromatin by RNA polymerase II: histone displacement and exchange. Mutat Res 618: 116-129, 2007.

4. Neely KE and Workman JL: The complexity of chromatin remodeling and its links to cancer. Biochim Biophys Acta 1603: 19-29, 2002.

5. Hake S, Xiao A and Allis C: Linking the epigenetic 'language' of covalent histone modifications to cancer. $\mathrm{Br} \mathrm{J}$ Cancer 90: 761-769, 2004.

6. Strahl BD and Allis CD: The language of covalent histone modifications. Nature 403: 41-45, 2000.

7. Peterson CL and Laniel MA: Histones and histone modifications. Curr Biol 14: R546-R551, 2004.

8. Redon C, Pilch D, Rogakou E, et al: Histone $\mathrm{H} 2$ a variants $\mathrm{H} 2 \mathrm{AX}$ and H2AZ. Curr Opin Genet Dev 12: 162-169, 2002.

9. Pehrson JR and Fried VA: MacroH2A, a core histone containing a large nonhistone region. Science 257: 1398-1400, 1992.

10. Kustatscher G, Hothorn M, Pugieux C, Scheffzek K and Ladurner AG: Splicing regulates NAD metabolite binding to histone macroH2A. Nat Struct Mol Biol 12: 624-625, 2005.

11. Brooks WH: X chromosome inactivation and autoimmunity. Clin Rev Allergy Immunol 39: 20-29, 2010.

12. Chadwick BP and Willard HF: Histone H2A variants and the inactive $\mathrm{X}$ chromosome: identification of a second macroH2A variant. Hum Mol Genet 10: 1101-1113, 2001. 
13. Kapoor A, Goldberg MS, Cumberland LK, et al: The histone variant macroH2A suppresses melanoma progression through regulation of CDK8. Nature 468: 1105-1109, 2010.

14. Rickert P, Seghezzi W, Shanahan F, Cho H and Lees E: Cyclin C/CDK8 is a novel CTD kinase associated with RNA polymerase II. Oncogene 12: 2631-2640, 1996.

15. Donner AJ, Szostek S, Hoover JM and Espinosa JM: CDK8 is a stimulus-specific positive coregulator of p53 target genes. Mol Cell 27: 121-133, 2007.

16. Sánchez I and Dynlacht BD: New insights into cyclins, CDKs, and cell cycle control. Semin Cell Dev Biol 16: 311-321, 2005.

17. Satyanarayana A and Kaldis P: Mammalian cell-cycle regulation: several Cdks, numerous cyclins and diverse compensatory mechanisms. Oncogene 28: 2925-2939, 2009.

18. Fisher RP: CDKs and cyclins in transition(s). Curr Opin Genet Dev 7: 32-38, 1997.

19. Kastan MB and Bartek J: Cell-cycle checkpoints and cancer. Nature 432: 316-323, 2004

20. Andreassi MG: DNA damage, vascular senescence and atherosclerosis. J Mol Med 86: 1033-1043, 2008.

21. Morgan DO: Cyclin-dependent kinases: engines, clocks, and microprocessors. Annu Rev Cell Dev Biol 13: 261-291, 1997.

22. Tyson JJ, Csikasz-Nagy A and Novak B: The dynamics of cell cycle regulation. Bioessays 24: 1095-1109, 2002.

23. Sherr CJ and Roberts JM: CDK inhibitors: positive and negative regulators of G1-phase progression. Genes Dev 13: 1501-1512, 1999.

24. Taylor WR and Stark GR: Regulation of the G2/M transition by p53. Oncogene 20: 1803-1815, 2001.

25. Sporn JC, Kustatscher G, Hothorn T, et al: Histone macroH2A isoforms predict the risk of lung cancer recurrence. Oncogene 28: 3423-3428, 2009.
26. Novikov L, Park JW, Chen H, et al: QKI-mediated alternative splicing of the histone variant MacroH2A1 regulates cancer cell proliferation. Mol Cell Biol 31: 4244-4255, 2011.

27. Haugstetter A, Loddenkemper C, Lenze D, et al: Cellular senescence predicts treatment outcome in metastasised colorectal cancer. Br J Cancer 103: 505-509, 2010.

28. Buschbeck M and Di Croce L: Approaching the molecular and physiological function of macroH2A variants. Epigenetics 5: $118-123,2010$.

29. Zhang R and Adams PD: Heterochromatin and its relationship to cell senescence and cancer therapy. Cell Cycle 6: 784-789, 2007.

30. Collado M, Gil J, Efeyan A, et al: Tumour biology: senescence in premalignant tumours. Nature 436: 642-642, 2005.

31. Dardenne E, Pierredon S, Driouch K, et al: Splicing switch of an epigenetic regulator by RNA helicases promotes tumor-cell invasiveness. Nat Struct Mol Biol 19: 1139-1146, 2012.

32. Shtutman M, Zhurinsky J, Simcha I, et al: The Cyclin D1 gene is a target of the beta-catenin/LEF-1 pathway. Proc Natl Acad Sci USA 96: 5522-5527, 1999.

33. Fu M, Wang C, Li Z, Sakamaki T and Pestell RG: Minireview: Cyclin D1: normal and abnormal functions. Endocrinology 145: 5439-5447, 2004.

34. Hunter T and Pines J: Cyclins and cancer II: Cyclin D and CDK inhibitors come of age. Cell 79: 573-582, 1994.

35. Gray N, Détivaud L, Doerig C and Meijer L: ATP-site directed inhibitors of Cyclin-dependent kinases. Curr Med Chem 6: 859-876, 1999

36. Lapenna S and Giordano A: Cell cycle kinases as therapeutic targets for cancer. Nat Rev Drug Discov 8: 547-566, 2009. 